Écrire

l'histoire

\section{Écrire l'histoire}

Histoire, Littérature, Esthétique

18 | 2018

Révolution

\title{
Quand les images d'histoire se donnent aux mots
}

I Am Not Your Negro de Raoul Peck

\section{Anne Crémieux}

\section{OpenEdition}

Journals

Édition électronique

URL : http://journals.openedition.org/elh/1549

DOI : $10.4000 /$ elh.1549

ISSN : 2492-7457

Éditeur

CNRS Éditions

\section{Édition imprimée}

Date de publication : 20 novembre 2018

Pagination : 227-231

ISBN : 978-2-271-12431-9

ISSN : 1967-7499

Référence électronique

Anne Crémieux, "Quand les images d'histoire se donnent aux mots », Écrire l'histoire [En ligne], 18|

2018, mis en ligne le 20 novembre 2018, consulté le 16 février 2021. URL : http://

journals.openedition.org/elh/1549; DOI : https://doi.org/10.4000/elh.1549 


\section{Quand les images d'histoire se donnent aux mots}

\section{Am Not Your Negro de Raoul Peck}

"History is not the past. It is the present. "

James Baldwin, « Black English. A Dishonest Argument (1980) I Am Not Your Negro (1: $22: 55)$

I Am Not Your Negro a d'abord fait une sortie très remarquée aux États-Unis en festivals, en musées et sur invitations universitaires, de la veille de l'élection de Donald Trump en septembre 2016 jusqu'en février 2017, avant de sortir en salles et en VOD. La présence fréquente, lors de ces projections-rencontres, de Raoul Peck, passionné de l'œuvre de Baldwin et incarnation de son influence et de sa postérité, a contribué au charisme de l'œuvre portée en anglais par la voix de Samuel L. Jackson et en français par celle de Joey Starr, tous deux choisis pour leur notoriété populaire. Je ne suis pas votre nègre est diffusé sur la chaîne Arte le 25 avril 2017, puis en salles de cinéma. On peut prédire au film un avenir pédagogique en France, terre d'accueil de Raoul Peck et pays coproducteur du film, et dans le monde ${ }^{1}$.
Alors que le mouvement\#BlackLivesMatter se poursuit et qu'une large minorité américaine a élu un homme dont la campagne qualifia les violences contre le mouvement des droits civiques comme appartenant au "bon vieux temps » (" the good old days ", rallye de campagne à Las Vegas, 22 février 2016), le message de Baldwin sur l'actualité de l'histoire résonne aux États-Unis et dans le monde entier, où les tensions ne font que se déplacer d'une communauté à l'autre en reproduisant des schémas similaires, schémas que la pensée de Baldwin propose de démêler par le retournement dialectique.

Les idées de Baldwin pourraient appartenir à l'histoire révolue de la lutte des années soixante. Cependant, ce n'est pas le cas, et ce pour trois raisons: parce que Baldwin a développé 
une analyse historique du racisme qui perdure aujourd'hui; parce que cette analyse possède une dimension psychosexuelle en avance sur son temps; et parce que Raoul Peck actualise la pensée de Baldwin par l'association d'images contemporaines et d'images historiques qui illustrent les voix mélangées de Baldwin et de ses interprètes modernes, lesquels reprennent mot pour mot le phrasé sophistiqué de l'écrivain homosexuel.

\section{Le racisme américain, hier et aujourd'hui}

À travers tout le film, Baldwin exhorte son auditoire à se poser la question qui découle de l'affirmation du titre et qui ne viendra véritablement qu'à la toute fin du film: si les Blancs ont inventé ce nègre (nigger, édulcoré en negro dans le titre) qui n'existe pas, dans lequel ni lui ni ses compatriotes ne se reconnaissent tel qu'il est présenté au cinéma, au journal télévisé ou dans les publicités, alors, que représente ce nègre ? Quel est son rôle $(1: 25: 20)$ ?

L'aveuglement des Blancs n'est jamais aussi clair que lorsque le philosophe Paul Weiss (l'onomastique ne vaut pas dans un documentaire, et pourtant...), invité à discuter sur un plateau de télévision $(1: 08: 07)$, reprend de façon si calmement condescendante les propos de Baldwin pour les présenter comme paranoïaques, obsédés par les questions raciales alors que la vraie question est que " chacun, face à lui-même, doit devenir un homme ". Baldwin tente de recadrer le débat vers ce sur quoi il porte : un Noir qui tente de devenir un homme met sa vie en danger. Weiss refuse cette « exagération » alors qu'il y a « tant d'autres façons de connecter les hommes». Baldwin, visiblement hors de lui, répond fort calmement, la verve crescendo, qu'il ne $\mathrm{s}^{\prime}$ agit pas de diviser les individus mais de comprendre, notamment quand on est blanc, qu'aucun individu n'a le pouvoir d'annuler le racisme institutionnel de l'Église, des agences immobilières, de l'armée, de l'école, des syndicats, et que donc, en effet, un homme noir américain est avant tout noir, quelles que soient les autres catégories qui pourraient le rapprocher d'hommes qui ne sont pas noirs en fonction d'un idéal fantasmé qui ne se traduit pas dans la réalité.

Lorsque Baldwin fait la liste des institutions de la ségrégation, le public de 2018 pense aux inégalités toujours réelles entre Noirs et Blancs, riches et pauvres, légaux et illégaux... En 2016, le mouvement \#BlackLivesMatter est à son apogée, qui dénonce les morts prématurées des personnes de couleur. Le reste du film propose les images qui permettent au public de faire ces liens, de répondre à la question finale du film, de faire face à ce que le professeur Weiss ne veut pas voir, au nom de l'humanisme. «On ne peut pas changer tout ce qu'on affronte, mais rien ne peut changer tant qu'on ne l'affronte pas" ("Not everything that is faced can be changed but nothing can be changed until it is faced", $1: 22: 40$ ). Ce que Baldwin souhaite que ses compatriotes affrontent, $c^{\prime}$ est le racisme institutionnel, mais c'est aussi, plus profondément, le rapport à l'Histoire, qui ne s'étudie pas au passé mais se porte au présent: "We carry our 
history with us. We are our history » $(1: 23$ : $00)$. Refuser de le reconnaître, c'est reproduire les crimes du passé ("If we pretend otherwise, we literally are criminals »).

La blancheur est pour Baldwin un concept indépendant des individus, qu'il n'a jamais pu haïr entièrement (il n'a pu rejoindre les Black Panthers), ayant compris au contact de Blancs anti-système, notamment une maîtresse d'école qui a soutenu son éducation

\section{Baldwin psychosociologue}

Les écrits de Baldwin contiennent de nombreuses références au cinéma et son essai de 1976, The Devil Finds Work, lui est entièrement consacré. Raoul Peck en reprend les exemples majeurs et, dans la partie intitulée " Heroes » $(11: 20)$, de longues citations illustrées: Baldwin y explique comment, petit, il comprit que les héros de westerns étaient ses ennemis (15: 34) et qu'il ne pouvait s'identifier qu'aux Indiens (16:48), l'héroïsme noir étant par ailleurs impossible au cinéma. Le héros noir hollywoodien garde toujours quelque chose de l'Oncle Tom, n'a pas de sexualité et ne reflète que rarement la réalité de la vie des Noirs, niée par une idéologie de la consommation heureuse. Raoul Peck poursuit par des images de grands magasins où l'abondance s'offre aux familles parfaites, des extraits d'émissions de game shows où les familles gagnent des produits de consommation pour leur plus grand plaisir, partagé par un public hystérique. Selon Baldwin, les Américains vivent dans un monde où toute connexion humaine est rendue impossible par le tabou du mélange des races et de l'homosexualité. politique et littéraire, que ce n'était pas la peau qui rendait les Blancs blancs, mais le pouvoir. "Le monde n'est pas blanc. Il ne l'a jamais été, ne peut pas l'être. Le blanc est une métaphore du pouvoir, juste une manière de décrire la Chase Manhattan Bank» $(1: 23: 00)$. Ce pouvoir, à l'heure du film de Peck, a peu changé de couleur - et quand bien même, il reste aveugle et/ou impuissant face au racisme institutionnel.

Bien qu'il l'évoque peu, I Am Not Your Negro n'oblitère pas entièrement la dimension queer de la théorie baldwinnienne. Le rapport du FBI (23:00) note l'homosexualité de ce dangereux personnage qui raconte ses vacances en République dominicaine avec « Lucien » (36: 55). Peck inclut les réflexions de Baldwin sur la fin de In the Heat of the Night (Dans la chaleur de la nuit de Norman Jewison, 1967), où il imagine un langoureux baiser entre le détective noir Sidney Poitier et le shérif blanc qui lui déclare son amitié sur le quai de la gare, conquis malgré ses préjugés racistes. Ces sentiments impossibles entre Blancs et Noirs, hommes ou femmes, sont pour Baldwin la manifestation d'un profond "échec de la sphère privée " ("failure of the private life», $44: 53$ ) qui se traduit autant qu'il est induit par la terreur acquise et construite à l'égard des populations noires, "une terreur sans fond ni nom qui se focalise sur le Noir comme figure d'effroi, sur une entité qui n'existe que dans son esprit [du Blanc] » (46:55). Peck, avec Baldwin, met en relation par le discours et l'image les tabous 
sexuels de l'Amérique et l'idéologie du rêve américain en illustrant ces propos par les scènes clés des problem films des années cinquante (No Way Out et The Defiant Ones) où Sidney Poitier se sacrifie pour son partenaire blanc à l'écran. Ces fantasmes sociétaux se manifestent aussi dans le héros asexué de Devine qui vient dîner (Stanley Kramer, 1967) ou à travers Godfrey Cambridge émergeant d'une banane pour chanter le slogan de «Chiquita Banana» (les Français penseront aux publicités pour Banania, ou même à Josephine Baker). Peck poursuit par un reportage sur le nouveau pouvoir d'achat des Noirs, qui, eux aussi, pourraient arpenter ces grands magasins filmés en grand angle, participer aux défilés de mode établissant des normes de beauté, prendre part aux game shows que Peck donne à voir et où

\section{Peck double Baldwin}

Le contraste permanent entre présent et passé se fait notamment par la juxtaposition d'images : la voix blanche qui raconte l'Amérique rêvée des années cinquante $(1: 04: 30)$ est illustrée par la statue de la Liberté, un match de baseball, un saut dans la piscine, un parc d'attractions, et, alors que le narrateur continue d'évoquer ce rêve partagé par tous, "ces opportunités illimitées », les images du reportage d'époque font place aux violences policières d'hier et d'aujourd'hui (57 : 40).

À de nombreuses reprises - Raoul Peck ayant eu la générosité d'accompagner son film de conférences en salle de cinéma -, le cinéaste rappelle que, à quelques exceptions près, chaque mot les Noirs sont présents. Pour Baldwin, ce bonheur artificiel est synonyme d'irresponsabilité et de mort d'une nation, de connexions manquées, comme cet amour pour une femme blanche dont il relate les contraintes lorsqu'il était bien jeune. Lorsqu'à l'université de Cambridge Baldwin s'offusque de la prophétie de Robert Kennedy selon laquelle dans quarante ans (soit en 2008 environ) un Noir pourrait devenir président, alors que sa propre famille irlandaise (arrivée aux États-Unis au xIX ${ }^{\mathrm{e}}$ siècle) a accès à la meilleure éducation et aux plus hautes fonctions de l'État, Baldwin conclut que l'Amérique vit dans un rêve, le rêve américain qui, parce qu'il exclut les Noirs, s'autodétruit (57 : 40). Pour Peck, les Noirs font désormais partie, eux aussi, de ce rêve qui continue de les détruire.

prononcé en voix off a été écrit par Baldwin. Mais le travail de chercheur, par la compilation d'une bibliographie que le cinéaste connaît sur le bout des doigts, le travail d'archiviste, rendu possible grâce au fait qu'il a eu accès à l'ensemble des lettres et des inédits de Baldwin, et le travail de documentariste, dont la clé principale est le montage, sont ce qui permet la narration. Alors que l'intrigue se résume à la réflexion politique autour de la lutte des Africains américains, $l^{\prime}$ on suit sans relâche la démonstration d'un Raoul Peck qui contrôle le récit à base d'écrits inachevés, remaniés, compilés et, enfin, illustrés.

Si Peck évoque de nombreux films chers à Baldwin (son premier souvenir 
cinématographique, Dance, Fools, Dance, son dédain pour Stepin Fetchit et autres Oncle Tom), il ne se prive pas d'illustrer ses critiques de la consommation à outrance par des images anachroniques de l'émission controversée de Jerry Springer (1991), ou les réflexions sur la criminalité systémique et la répression policière et judiciaire par des extraits d'Elephant (Gus Van Sant, 2003). Le documentariste alterne donc illustration directe et illustration métaphorique. Il relaie également les images d'archives contemporaines $\mathrm{du}$ discours de Baldwin (par exemple celles des meurtres de Malcolm X (1965) et de Martin Luther King (1968) ; de l'annonce de ce deuxième meurtre par Robert Kennedy, assassiné quelques mois plus tard; de l'entrée dans un lycée blanc de Dorothy Counts en 1957) par celles du passage à tabac de Rodney King, des manifestations \#BlackLivesMatter et de la répression policière par des hommes mieux protégés et mieux armés que les policiers violents des années cinquante et soixante. Lorsque Baldwin évoque les meurtres de jeunes Noirs (41: 10), Peck

\section{Note}

1 Voir James BALdwin, Raoul PECK, I Am Not Your Negro, New York, Vintage Books, 2017, traduit en français la même année sous le même titre chez R. Laffont ; Toni Pressley-SAnon, Sophie illustre ses paroles par les portraits de jeunes filles et garçons où s'affichent leurs dates bien trop rapprochées de naissance et de mort au xxI ${ }^{\mathrm{e}}$ siècle, puis une photo en noir et blanc de manifestants dénonçant les meurtres de l'État policier, une manifestation sans âge: de l'époque du discours que l'on entend ou du film que l'on regarde? C'est incertain. Le blues de la bande son fait aussi le lien poignant entre les époques.

L'écriture filmique de la pensée historique se fait par une multitude d'images qui défilent trop vite pour être toutes clairement placées et distinctement reliées aux paroles de Baldwin. L'intelligence du film tient à celle qu'il suppose au grand public, invité à revoir non seulement sa réflexion sur les relations raciales et l'histoire de l'Amérique et de l'Occident, mais aussi son rapport à l'image et à l'idéologie qu'elle véhicule. Est-ce être, comme Baldwin, optimiste que de prédire que cette mise en images d'une parole provocatrice sera encore longtemps servie par les injustices constitutives des sociétés humaines?
SaInt-Just (dir.), Raoul Peck. Power, Politics, and the Cinematic Imagination, Lanham (Md.), Lexington Books, 2015. 